\title{
Watching worms grow, cell by cell
}

Caenorhabditis elegans is a well-established model organism first introduced to the laboratory in the 1960 s by Sydney Brenner. Comprised of just 959 cells and maturing in only a few days, these small, transparent nematodes have provided researchers with unique insights into developmental biology. Cell fates have been mapped, but observing all of the transitions from embryo to adult has been challenging because worms are, frankly, squirmy. Images of freely moving larvae can be taken but have lacked singlecell detail, while acquiring high-resolution images requires that the animals be immobilized. Mechanical restriction and paralytic drugs can hold them still long enough to take a decent picture, but prevent feeding. In an organism with such a short life cycle, even minimal disruptions can quickly arrest further development. Visualizing C. elegans at the cellular level over the full course of its development was hypothetically possible, but in need of an improved approach.

To resolve this imaging conundrum, Dutch researchers led by Jeroen van Zon at
FOM Institute AMOLF in Amsterdam have developed a new quantitative microscopy technique for tracking C. elegans development (Nat. Commun. 7, 12500; 2016). The team manufactured a $10 \times 10$ array of microchambers filled with a polyacrylamide hydrogel, which slows the worms down without completely stopping them from moving about. A single embryo was placed in each chamber along with some E. coli for food. LED illumination, short exposure times, and high numerical aperture allowed the researchers to take timelapse microscopic photographs of the entire chamber while resolving each individual cell. Their images corresponded with established markers such as molting episodes and body length extension-indicating that the set-up did not impede developmentyet allowed them to document individual variation for 10 to 20 worms simultaneously every 20 minutes over 48 hours as they matured from embryos to adults.

The researchers further demonstrated the utility of their microscopy technique

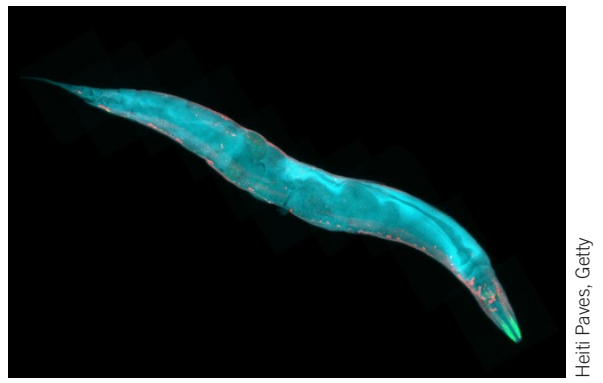

by following cell division, cell migration, and gene expression dynamics in wildtype C. elegans as well as various mutants, with the help of fluorescent tags. Each of the processes examined occurs over timeframes too long to be adequately captured by previous techniques but could be suitably imaged in the current study, offering novel insight into C. elegans development. The team hopes that their microscopy technique will be a valuable tool for studying C. elegans in future research.

Ellen P. Neff

\section{ZEBRAFISH AS MODELS OF MICROBE-MODULATED ANXIETY}

New research continues to add evidence for the influence of microbiota on an organism's health and behavior. Researchers are probing what is known as the microbiota-gut-brain axis, an apparent connection between microbes in the gut and the central nervous system. The communication is thought to be two-way, but elucidating the exact mechanisms is difficult given the myriad microorganisms out there, physiological differences between hosts, and challenges in tracking neuronal signals. Germ-free mice and other gnotobiotic varieties are increasingly under review, but developing alternative models in other species can provide novel opportunities to study the workings of the microbiota-gut-brain axis.

Zebrafish are proving to be popular candidates. Neurobehavioral phenotypes have been documented that correspond well to those established in mice, and they share some characteristics with humans, such as cortisol production under stress, that differ in rodents. Their use in high-throughput screening is also well established, which could speed drug testing if new targets for treating diseases or conditions are identified. In the context of microbial studies, zebrafish are uniquely valuable: they are transparent, enabling internal visualizations if researchers introduce labeled microbes, and they develop from eggs, making it easier to manipulate their innate microbial profiles. A new research article (Behav. Brain Res. 311, 219-227; 2016) and related metagenomic analysis (Data Brief 8 , 938-943; 2016) highlight the use of zebrafish in microbial studies.

In particular, the researchers were interested in the relationship between the microbiome and anxiety during early development, the results of which are thought to have lasting effects into adulthood. They examined measures of anxiety in three groups of larval zebrafish: germ-free, which were carefully washed after fertilization with a series of antibiotic, iodine, and bleach solutions before being housed in sterilized water; conventionalized, which underwent the sterilization process but were held in standard fish water; and conventionally raised, receiving no special treatment. Each group had different microbial constituents (with germ-free zebrafish harboring none); despite the differences, conventionalized and conventionally raised fish responded similarly to subsequent tests. Germ-free zebrafish were considerably more carefree than germy ones. They displayed less thigmotaxis, or 'wall-hugging', during an activity assay and did not produce increased cortisol in response to an osmotic stress test. A splash of the probiotic Lactobacillus plantarum, however, calmed anxious behavior in the conventionally raised larvae.

These results demonstrate a link between microbiota and anxiety in zebrafish larvae, as has been previously documented in mice. They also establish zebrafish as a useful alternative model to rodents for consideration in future studies of the microbiota-gut-brain axis.

Ellen P. Neff 